\title{
Primary central nervous system lymphoma presenting with Parkinsonism as an initial manifestation: A case report and literature review
}

\author{
RINTARO OKANO, KOHEI SUZUKI, YOSHITERU NAKANO and JUNKOH YAMAMOTO
}

Department of Neurosurgery, University of Occupational and Environmental Health, Kitakyushu, Fukuoka 807-8555, Japan

Received July 27, 2020; Accepted February 5, 2021

DOI: $10.3892 / \operatorname{mco} .2021 .2257$

\begin{abstract}
Primary central nervous system lymphoma(PCNSL) is a rare intracranial neoplasm in older adults. Tumor-associated parkinsonism (TAP) in PCNSL is extremely rare, and its clinical features are unclear. The present report describes the case of a 75-year-old man who presented with parkinsonism due to multiple hyperintense lesions in the thalamus and periventricular white matter as visualized by magnetic resonance imaging (MRI). Due to the rapid progression of parkinsonism and lesion enlargement, the patient underwent stereotaxic biopsy. Subsequently, his condition was diagnosed as TAP in PCNSL at 2 months after onset. The patient completely recovered after treatment and experienced no recurrence of TAP for 8 months. Although it is difficult to distinguish TAP from vascular parkinsonism (VP) at initial consultation, the early diagnosis of PCNSL is important for improving prognosis. In the case of rapidly progressing parkinsonism, one should suspect the possibility of TAP associated with early-stage PCNSL. Early treatment improves the chances of remission and decreases the possibility of recurrence.
\end{abstract}

\section{Introduction}

Primary central nervous system lymphoma (PCNSL) is a rare intracranial neoplasm. Although the incidence of PCNSL

Correspondence to: Dr Kohei Suzuki, Department of Neurosurgery, University of Occupational and Environmental Health, 1-1 Iseigaoka, Yahatanishi-ku, Kitakyushu, Fukuoka 807-8555, Japan E-mail: s-kohei@clinc.uoeh.ac.jp

Abbreviations: PCNSL, primary central nervous system lymphoma; TAP, tumor-associated parkinsonism; VP, vascular parkinsonism; MRI, magnetic resonance imaging; FLAIR, fluid-attenuated inversion recovery; CT, computed tomography; DAT SPECT, dopamine transporter single photon emission computed tomography; KPS, Karnofsky performance status; DLBCL, diffuse large B-cell lymphoma; PD, Parkinson's disease; CI, cerebral infarction; T1WI, T1 weighted image; T2WI, T2 weighted image

Key words: PCNSL, TAP, secondary parkinsonism, VP, thalamic lesion has increased in patients over 60 years of age in the past two decades; it currently accounts for a mere $2.4-4.5 \%$ of all newly diagnosed brain tumors $(1,2)$. PCNSL is an uncommon subtype of extranodal non-Hodgkin lymphoma that involves the central nervous systems, and the majority of tumors are B-cell phenotypic origin. The risk factor for the development of PCNSL is immunodeficiency, however, there are also seems to be increase in incidence of PCNSL in immunocompetent individuals (2). PCNSL initially presents with various clinical symptoms, such as focal neurological deficits, neuropsychiatric symptoms, increased intracranial pressure, and convulsive seizures (3). Despite the varied clinical presentation, parkinsonism is extremely rare as an initial clinical symptom of PCNSL.

Here, we report a rare case of PCNSL presenting with parkinsonism as an initial clinical symptom in an older adult, and emphasize the difficulty in distinguishing tumor-associated parkinsonism (TAP) from vascular parkinsonism (VP) during the initial diagnosis.

\section{Case report}

A 75-year-old man without any history of immunocompromise was referred to our hospital due to bradykinesia, discrete movement disorder, and low speech for a month prior to referral. Before admission, the patient developed a broad base gait disturbance and a pill-rolling tremor, both of which were evident upon examination at the time of admission. (Fig. 1). Magnetic resonance imaging (MRI) with fluid-attenuated inversion recovery (FLAIR) showed multiple hyperintense lesions in the left basal ganglia, thalamus, and bilateral frontal periventricular white matter (Fig. 2A and B). A computed tomography (CT) showed low-density lesions in the bilateral periventricular white matter and a slightly hyperdense lesion in the left thalamus (Fig. 2C). A dopamine transporter single photon emission computed tomography (DAT SPECT) showed a decrease in ${ }^{123} \mathrm{I}$-Ioflupane uptake in the left striatum (Fig. 2D). Considering the chronic ischemic changes suspected from the MRI and DAT SPECT findings, we classified his condition as VP and discharged the patient with frequent follow-up instructions. One month later, the patient's condition further deteriorated, presenting with impairment of the postural reflex, a mask-like face, and micrographia. Due to the rapid progression of VP, he underwent reexamination by MRI, revealing enlargement of the hyperintense lesions in 
75 yearold nan without nedical history

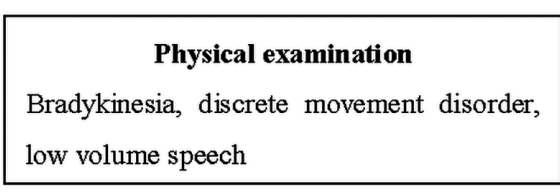

Gait disturbance, pill-rolling tremor

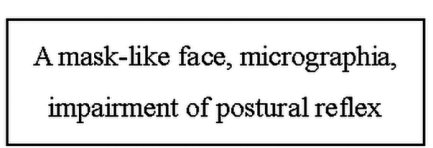

Completely recovered from parkinsonism

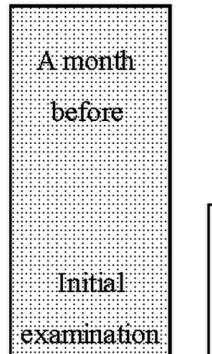

MRI Multiple hyperintense lesions in the left basal ganglia, thalamus, bilateral frontal PWM on FLAIR

DAT SPECT a decrease in ${ }^{123} I$-Ioflupane uptake in the left striatum

Diagnosed as a vascular parkinsonism

MRI Enlargement of the hyperintense lesions in the left thalamus and frontal PWM
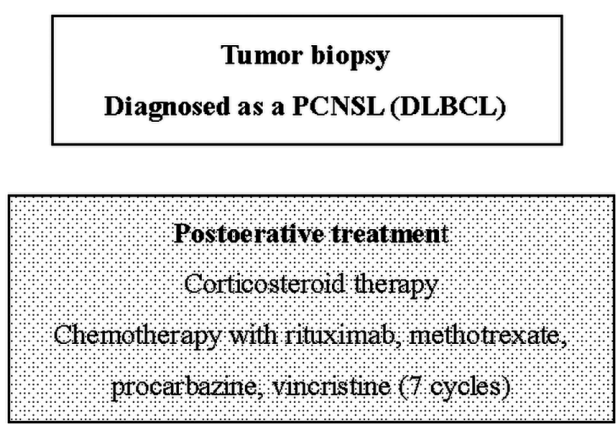

procarbazine vincristine $(\mathrm{cycles})$
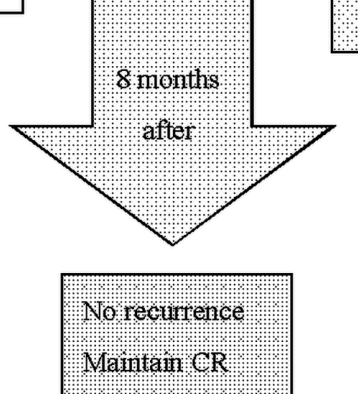

Figure 1. Timeline of the patient's clinical course. MRI, magnetic resonance imaging; DAT SPECT, dopamine transporter single photon emission computed tomography; PWM, periventricular white matter; FLAIR, fluid-attenuated inversion recovery; PCNSL, primary central nervous system lymphoma; DLBCL, diffuse large B cell lymphoma; CR, complete response.

the left thalamus and frontal periventricular white matter with heterogeneous enhancement (Fig. 2E-H). He was subsequently admitted to our department, with a Karnofsky Performance Status (KPS) at admission of 40. Serological examinations showed mildly elevated interleukin-2 receptor levels $(657 \mathrm{U} / \mathrm{ml})$ and normal levels of lactate dehydrogenase (161 U/l). His HIV antibody test results were negative. Because of the rapid and atypical progression of the disease, we suspected TAP due to the brain lesion and performed a stereotactic biopsy for the left frontal periventricular lesion two months after onset of symptoms. The pathological examination revealed proliferation of medium- to large-sized atypical lymphoid cells around the small vessels. These tumor cells strongly expressed CD20, corresponding to a diffuse large B-cell lymphoma (DLBCL) (Fig. 3). A genetic analysis was not performed due to limitations in the examination. Given this report, we diagnosed his condition as TAP in DLBCL, and he immediately underwent corticosteroid therapy. His condition rapidly improved with treatment, and his KPS immediately after initial treatment improved to 70 . He then underwent multidrug chemotherapy with rituximab, methotrexate, procarbazine, and vincristine two weeks after surgery. One month after treatment, the patient had completely recovered from TAP and was discharged without any neurological deficits. Follow-up MRI without contrast enhancement (four months after treatment) revealed that the lesion in the left thalamus had disappeared and the periventricular lesion had shrunk (Fig. 4A-D). He received seven cycles of chemotherapy, and ultimately achieved complete response. Eight months after treatment, his KPS remained at 70, and he had no recurrence of TAP. 
A

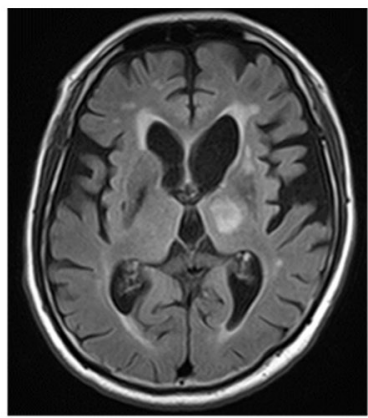

E

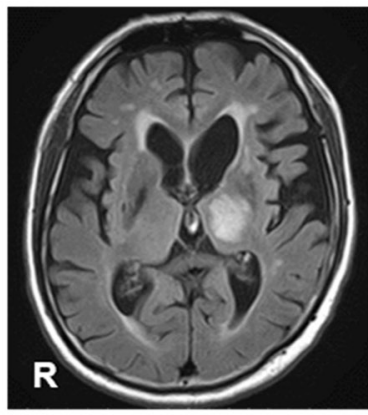

B

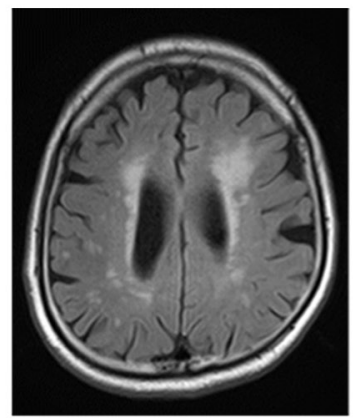

F

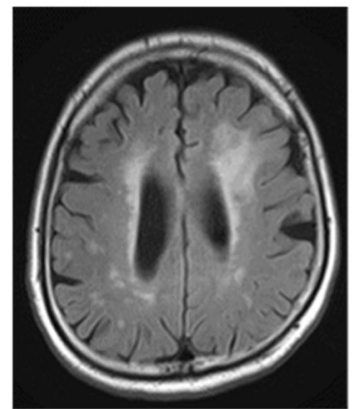

c

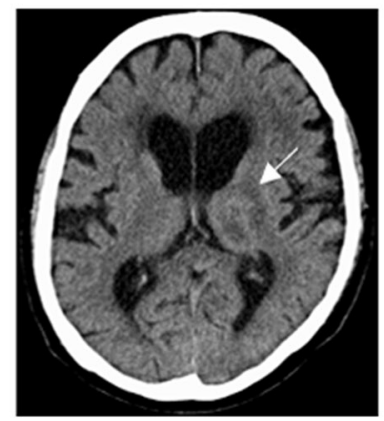

G

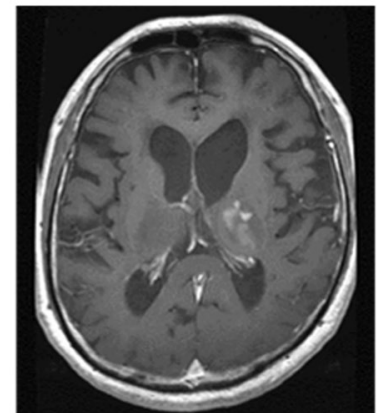

D

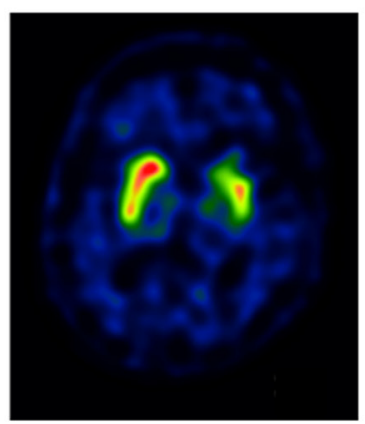

H

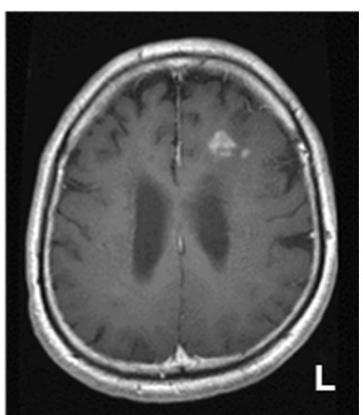

Figure 2. Pretreatment radiological findings. Initial axial FLAIR revealed disruption of (A) the left basal ganglia and (B) bilateral frontal periventricular white matter. (C) Axial computed tomography revealed a slight high-density lesion in the left thalamus (arrow). (D) Dopamine transporter single photon emission computed tomography revealed decreased ${ }^{123}$ I-Ioflupane uptake in the left striatum, in accordance with lesions observed on magnetic resonance imaging. Follow-up axial FLAIR (1 month after initial examination) revealed enlargement of the lesions in (E) the left basal ganglia and (F) frontal periventricular white matter. Axial contrast-enhanced T1 weighted images revealed a heterogeneous enhancement pattern in $(\mathrm{G})$ the left thalamus and (H) periventricular white matter. FLAIR, fluid-attenuated inversion recovery.

\section{A}

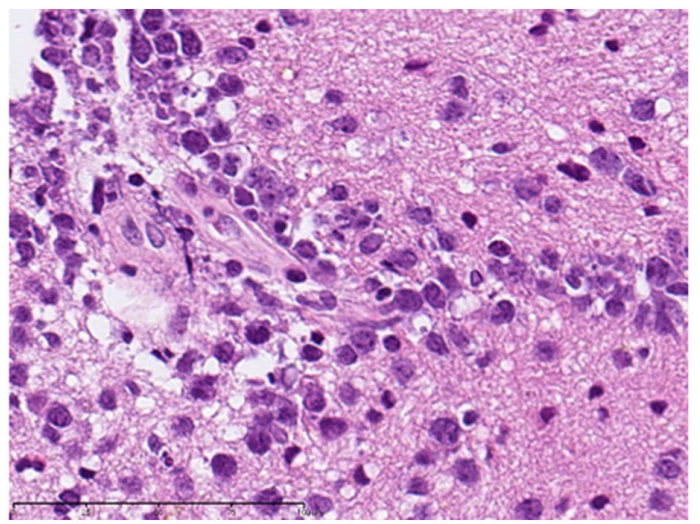

B

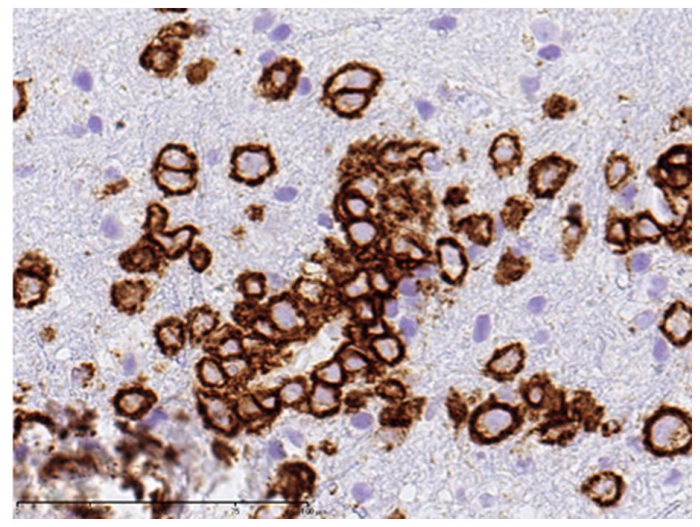

Figure 3. H\&E staining of tumor cells and immunohistochemical staining of CD20. (A) H\&E staining revealed proliferation of medium to large-sized atypical lymphoid cells with hyperchromatic nuclei and prominent nucleoli around the small vessels. (B) Immunohistochemical staining revealed CD20 expression in the atypical lymphocytes. Scale bar, $100 \mu \mathrm{m}$. H\&E, hematoxylin-eosin.

\section{Literature review}

PCNSL presents various clinical symptoms, such as focal neurologic deficit in $70 \%$ of patients, behavioral neuropsychiatric changes in $43 \%$, headache, nausea, and vomit associated with elevated intracranial pressure in $33 \%$, and seizures in $14 \%$ (3). However, TAP in PCNSL is extremely rare, and only 5 cases, including ours, have been reported so far (Table I) (4-7). TAP in PCNSL is relatively common in older patients, with a mean age at diagnosis of 74.2 (range, 64-81) years, and no sex preference, with three male and two female patients. Several clinical symptoms associated with parkinsonism were described. All patients presented with bradykinesia and gait disorder, followed by rest tremor, rigidity and fixed face in $60 \%$, impairment of the postural reflex in $40 \%$, and abnormal posture in only one case at initial treatment. Three patients $(60 \%)$ demonstrated laterality of clinical symptoms; however, two cases (40\%) presented with bilateral symptoms. Most cases presented with lesions at multiple sites, including the basal ganglia in $80 \%$ (putamen and globus pallidus in $60 \%$, thalamus in $20 \%$ ), the periventricular white matter in $40 \%$, and the corpus callosum in $20 \%$ of lesions. Only one case had a single lesion in the midbrain. Levodopa, which was started as initial treatment in three cases $(60 \%)$, was not effective. As TAP is a rare clinical symptom and course of PCNSL, and because precise diagnosis is typically 


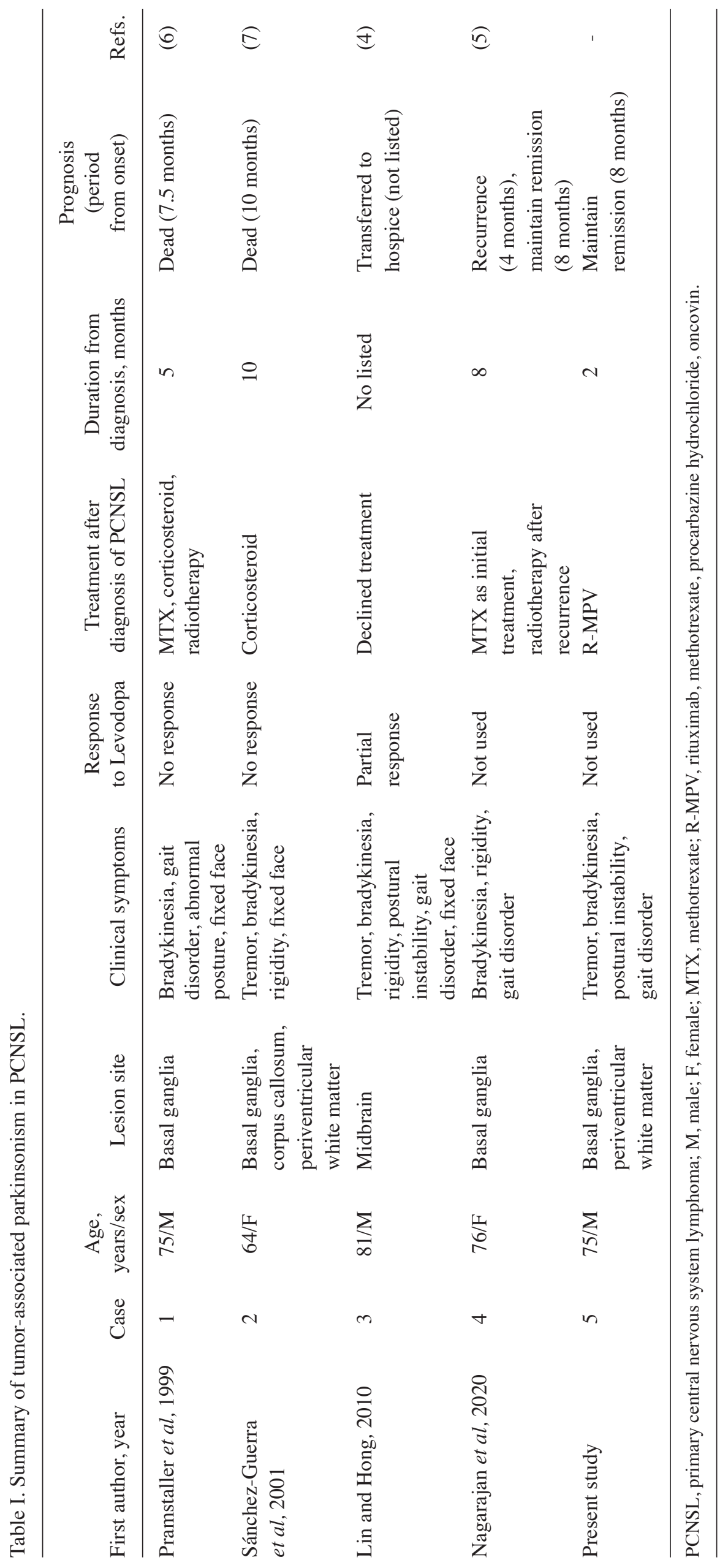


A

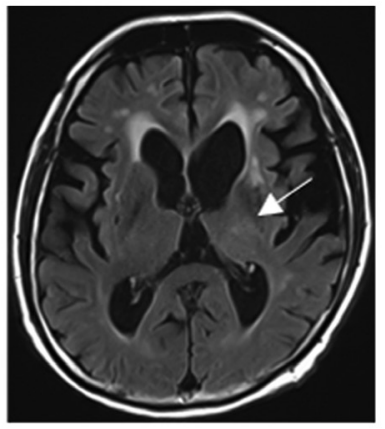

B

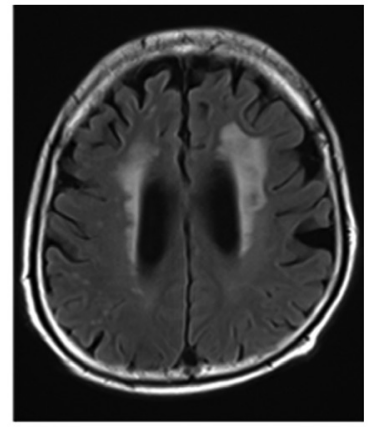

C

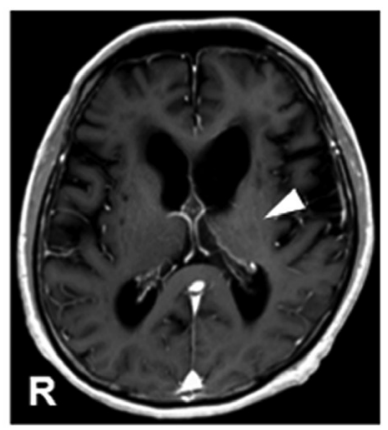

D

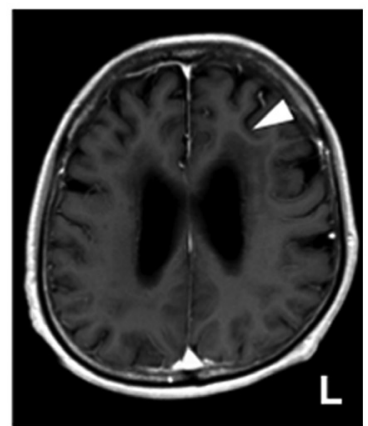

Figure 4. Follow-up magnetic resonance imaging 4 months after treatment. Axial fluid-attenuated inversion recovery revealed that the enlarged lesions in (A) the left thalamus had disappeared (arrow), whereas (B) the periventricular lesion was irreversible. Axial contrast enhanced T1 weighted images revealed heterogeneous enhancement in both $(\mathrm{C})$ the left thalamic lesions and (D) the periventricular white matter lesions that disappeared (arrow head).

delayed (duration: Over seven months), the prognosis is poor in most cases.

The first case of brain tumor with parkinsonism was reported in 1953, after then, several cases of TAP, which caused by meningioma, glioma, cavernoma, and other tumors, have sporadically been reported (8-11). Whereas, to the best of our knowledge, there are only 5 cases of TAP in PCNSL in the literatures as previously mentioned. Therefore, those clinical features are not well known, and TAP in PCLNS may be underestimated, clinicians should be aware of the risk of TAP in PCNSL.

\section{Discussion}

The clinical features of TAP in PCNSL are not well known because of their rare nature; however, it is important for diagnosis and treatment. Parkinson's disease (PD), which presents as bradykinesia with one additional symptom, such as rigidity, resting tremor, and postural instability, are well known. These tend to be unilateral initially, and present as persistent asymmetrical symptoms. In the beginning, symptoms are evident on one side of the body with the contralateral symptoms appearing within a few years (12). A previous report also described the clinical features of VP. The predominant manifestation is described as lower body parkinsonism, including gait disturbance and postural instability, which is similar to that observed in patients with PD. The progression of symptoms is variable in VP with an acute onset in some cases. These clinical symptoms commonly remain stable or deteriorate slowly (13). However, TAP in PCNSL progresses rapidly compared to $\mathrm{PD}$ and $\mathrm{VP}$.

In the present case, the initial manifestation was bradykinesia and low voice. As parkinsonism progressed, clumsiness, gait disturbance, and pill rolling movement appeared within month after initial onset. Unilateral symptoms were observed in only three cases, and may be associated with multiple lesions at diagnosis in most cases. In addition, diurnal fluctuations of symptoms were not observed in the present case, which may have led to the distinction between PD and TAP. Levodopa was not administered in the present case. Generally, an excellent response to levodopa is a supportive prospective criterion in PD (12). However, it has been demonstrated to be ineffective or only partially responsive in other TAP cases. Thus, in the case of rapid progression of parkinsonism or ineffective levodopa, we should suspect the possibility of TAP of PCNSL in older patients with basal ganglia lesions.

It is also challenging but critical to distinguish between cerebral infarction (CI) and PCNSL in the early stages of disease. CI commonly occurs in the basal ganglia, including the thalamic area. In particular, older adult patients develop chronic cerebral ischemia in the white matter of the cerebrum, including the thalamus, leading to VP (13). In contrast, brain tumors, such as gliomas, germinomas, and teratomas, rarely occur and are localized to the thalamic area. Although PCNSL commonly develops in the periventricular region, white matter, and corpus callosum, 17\% of PCNSLs are found in the thalamus and basal ganglia $(14,15)$. It may be difficult to distinguish between PCNSL and CI of localized lesions in the thalamus using conventional MRI without contrast reagent. Generally, PCNSL presents as iso- or hypointense on T1-weighted image (T1WI) and iso- or hyperintense on T2-weighted image (T2WI)/FLAIR images (15). Chronic CI, including lacunar and white matter lesions are hyperintense on T2WI/FLAIR in ordinary cases. Although CE-T1WI depicts the characteristic homogenous enhancement on PCNSL, contrast-enhanced imaging is not necessarily used as a routine examination in cases suspected of CI at primary screening. However, the PCNSL lesion presents as slightly iso- or hyperdense on CT, which is related to a very high nuclear/cytoplasmic ratio, in contrast with $\mathrm{CI}$ lesions that show as hypodense (16). In our case, MRI revealed a localized hyperintensity in the thalamus on FLAIR, thus leading to the initial diagnosis of VP. Retrospectively, initial CT showed an iso- to slightly hyperdense thalamic lesions; thus, we should have suspected the possibility of PCNSL. While rare, we suggest that PCNSL should be considered as a differential diagnosis in localized thalamic lesions in older adults, even though it presents in.

The mechanism of TAP in PCNSL remains unclear. Brain tumors presenting with TAP occur in various locations, such as the brain stem, basal ganglia, frontal lobe, temporal lobe, cerebellum, hypothalamus, sella, and pineal regions (8,9,17-19). Generally, PD is associated with cell loss in the substantia nigra, whereas only $31 \%$ of brain lesions presenting with parkinsonism include the substantia nigra (20). Brain tumors disrupt the neuronal circuits, such as the axons of the presynaptic dopaminergic neuron and the output pathway from postsynaptic cells of the basal ganglia circuit to the cortex, either by the mass effect of the tumor itself or by peritumoral brain edema, leading to the development of parkinsonism (21). In PCNSL, tumor cells invade and damage the neuronal membranes in the basal ganglia, resulting in the development of TAP (22). In the 
present case, PCNSL located in the periventricular white matter, basal ganglia, and thalamus, and ${ }^{123}$ I-Ioflupane SPECT showed decreased ${ }^{123}$ I-Ioflupane uptake in the left basal ganglia, which then developed TAP. Generally, PD causes distinctive neuropathological brain changes, such as the formation of abnormal proteinaceous spherical bodies called Lewy bodies, and drug response becomes limited with disease progression (12). However, TAP recovered following treatment for PCNSL. Thus, PCNSL induces reversible dysfunction of neuronal circuits in the thalamus, basal ganglia, and cortex of the cerebrum and midbrain during invasion within the perivascular space, triggering the development of TAP. Due to the high tumor proliferation of PCNSL, TAP progresses rapidly. Although a precise diagnosis of TAP may be difficult at initial diagnosis, TAP should be suspected and diagnosed as early as possible, and appropriate treatment for tumors should be administered before the physical manifestations of TAP become irreversible.

In conclusion, Parkinsonism is rarely an initial manifestation of PCNSL. In contrast, VP is common among older adults. Due to its rarity, it is often challenging to distinguish between TAP and VP at initial consultation. TAP should be suspected if the clinical symptoms of parkinsonism progress rapidly, and thus, physicians should consider repeat MR examination under close observation in these cases.

\section{Acknowledgements}

Not applicable.

\section{Funding}

No funding was received.

\section{Availability of data and materials}

The datasets used and/or analyzed during the current study are available from the corresponding author on reasonable request.

\section{Authors' contributions}

$\mathrm{RO}$ and KS drafted the manuscript and wrote the final paper. YN and JY made substantial contributions to conception and design. YN and JY drafted parts of the manuscript, revised the content critically and provided constructive feedback. KS and YN performed the surgery. RO and KS analyzed all images. KS and JY confirmed the authenticity of all the raw data. All authors read and approved the final manuscript.

\section{Ethics approval and consent to participate}

Not applicable.

\section{Patient consent for publication}

Oral and written informed consent was obtained from the patient for the publication of the case details and any associated images.

\section{Competing interests}

The authors declare that they have no competing interests.

\section{References}

1. Schlegel U: Primary CNS lymphoma. Ther Adv Neurol Disord 2: 93-104, 2009.

2. Villano JL, Koshy M, Shaikh H, Dolecek TA and McCarthy BJ: Age, gender, and racial differences in incidence and survival in primary CNS lymphoma. Br J Cancer 105: 1414-1418, 2011.

3. Grommes $C$ and DeAngelis LM: Primary CNS lymphoma. J Clin Oncol 35: 2410-2418, 2017.

4. Lin CM and Hong K: Cerebral infratentorial large B-cell lymphoma presenting as Parkinsonism. Tohoku J Exp Med 220: 187-190, 2010.

5. Nagarajan E, Yerram SY, Digala LP and Bollu PC: Primary central nervous system lymphoma presenting as parkinsonism with atypical MRI findings and elevated 14-3-3 protein. J Neurosci Rural Pract 11: 492-494, 2020.

6. Pramstaller PP, Salerno A, Bhatia KP, Prugger M and Marsden CD: Primary central nervous system lymphoma presenting with a parkinsonian syndrome of pure akinesia. J Neurol 246: 934-938, 1999.

7. Sánchez-Guerra M, Cerezal L, Leno C, Diez C, Figols J and Berciano J: Primary brain lymphoma presenting as Parkinson's disease. Neuroradiology 43: 36-40, 2001.

8. Al-Janabi WSA, Zaman I and Memon AB: Secondary parkinsonism due to a large anterior cranial fossa meningioma. Eur J Case Rep Intern Med 6: 001055, 2019.

9. Connolly ID, Johnson E, Lummus S and Hayden Gephart M: Massive intradural chondroma masquerading as lower body parkinsonism. Cureus 10: e2099, 2018.

10. Ertan S, Benbir G, Tanriverdi T, Alver I and Uzan M: Parkinsonism caused by cavernoma located in basal ganglion. Parkinsonism Relat Disord 11: 517-519, 2005.

11. Margulies ME: Parkinsonism and brain tumor. J Nerv Ment Dis 117: 550-552, 1953.

12. Sveinbjornsdottir S: The clinical symptoms of Parkinson's disease. J Neurochem 139: 318-324, 2016.

13. Korczyn AD: Vascular parkinsonism-characteristics, pathogenesis and treatment. Nat Rev Neurol 11: 319-326, 2015.

14. Linn J, Hoffmann LA, Danek A and Brückmann H: Differential diagnosis of bilateral thalamic lesions. Rofo 179: 234-245, 2007 (In German).

15. Renard D, Castelnovo G, Campello C, Bouly S, Le Floch A, Thouvenot E, Waconge A and Taieb G: Thalamic lesions: A radiological review. Behav Neurol 2014: 154631, 2014.

16. Haldorsen IS, Espeland A and Larsson EM: Central nervous system lymphoma: Characteristic findings on traditional and advanced imaging. AJNR Am J Neuroradiol 32: 984-992, 2011.

17. Choi KH, Choi SM, Nam TS and Lee MC: Astrocytoma in the third ventricle and hypothalamus presenting with parkinsonism. J Korean Neurosurg Soc 51: 144-146, 2012.

18. Duron E, Lazareth A, Gaubert JY, Raso C, Hanon O and Rigaud AS: Gliomatosis cerebri presenting as rapidly progressive dementia and parkinsonism in an elderly woman: A case report. J Med Case Rep 2: 53, 2008.

19. Yasuhara T, Agari T, Kambara H, Ichikawa T, Kurozumi K, Ono S, Miyoshi Y and Tokunaga K; Isao Date: Parkinsonism related to brain tumors: A case report and review of the literature. Open Neurosurgery J 2: 4-7, 2009.

20. Joutsa J, Horn A, Hsu J and Fox MD: Localizing parkinsonism based on focal brain lesions. Brain 141: 2445-2456, 2018.

21. Kim JI, Choi JK, Lee JW and Hong JY: Intracranial meningioma-induced parkinsonism. J Lifestyle Med 4: 101-103, 2014.

22. Merrill S, Mauler DJ, Richter KR, Raghunathan A, Leis JF and Mrugala MM: Parkinsonism as a late presentation of lymphomatosis cerebri following high-dose chemotherapy with autologous stem cell transplantation for primary central nervous system lymphoma. J Neurol 267: 2239-2244, 2020. 